\title{
COMMENTARY
}

\section{Mitochondrial DNA Mutations and Cancer}

\section{Lessons from the Parathyroid}

\author{
Konstantin Popadin, ${ }^{*}$ Konstantin V. Gunbin, ${ }^{\dagger}$ and Konstantin Khrapko ${ }^{\ddagger \S}$
}

From the University of Geneva Medical School, * Geneva, Switzerland; the Institute of Cytology and Genetics, ${ }^{\dagger}$ Novosibirsk, Russia; and the Departments of Biology $y^{\ddagger}$ and Pharmaceutical Sciences, ${ }^{\S}$ Northeastern University, Boston, Massachusetts

The article by Müller-Höcker et $\mathrm{al}^{1}$ in this issue of The American Journal of Pathology explores mitochondrial DNA (mtDNA) mutations of benign hyperplastic nodules of the hyperfunctional parathyroid gland. The study compares mtDNA mutations in cells differing by the extent of mitochondrial proliferation (ie, clear chief cells, pre-oxyphil, and oxyphil cells). Mitochondrial proliferation in these cells is thought to be a compensatory effect caused by a defect in respiratory chain. The transition from clear chief to oxyphil cells is an example of the oncocytic transformation, which is also observed in other tissues.

Müller-Höcker et al ${ }^{1}$ compare mtDNA mutations in normal cells with mutations in cells defective in cytochrome c oxidase (COX). COX, a key enzyme of the respiratory chain, is partially encoded by the mtDNA. Of particular importance is their discovery of substantial load of COX-inactivating mtDNA mutations and their conclusion that oncocytic transformation in the parathyroid gland can be caused by COX mutations. This conclusion extends an existing view that oncocytic transformation is caused almost exclusively by mutations in another respiratory chain enzyme partially encoded by mtDNA [ie, NADH dehydrogenase (ND)]. Although appearing too specialized, the impact of the highlighted study is quite broad as oncocytic cells give rise to oncocytic tumors, which are a rare example of tumors where involvement of mtDNA mutations is certain. This study may thus provide one of the missing tiles in the complicated puzzle depicting the role of mtDNA mutations in carcinogenesis.

\section{mtDNA Mutations in Tumors: Drivers or Drifters, Enemies or Friends?}

The question whether mutations in mtDNA may be involved in carcinogenesis has a long history of controversy. Perhaps the most influential discovery was that tumors tend to contain mtDNA mutations that are not found in the surrounding normal tissue. ${ }^{2}$ Because there are hundreds of mtDNA molecules in each cell, mutant mtDNA may be present at various fractions of the total mtDNA (a condition called heteroplasmy) up to $100 \%$ (homoplasmy). Strikingly, mtDNA mutations were not just present in tumors, but they constituted a high percentage ( $>50 \%$ ) heteroplasmy or even homoplasmy. Thus mitochondrial genomes carrying these mutations somehow had managed to out-compete their wildtype counterparts within tumor cells or tumor precursors, but not in normal tissue. Initially, this observation was perceived as evidence of dramatic positive selection of mutant mitochondrial genomes in tumors, implying that mutations were essential for carcinogenesis. However, later it was shown that somatic mtDNA mutations may reach homoplasmy in tumors by a purely random process similar to fixation of mutations in a population by genetic $\mathrm{drift}^{3}{ }^{3}$ Similar processes also operate in normal cells. ${ }^{4,5}$ Thus the mere presence, high incidence, or high fraction mtDNA mutations in tumors by itself cannot be used as evidence of their involvement in carcinogenesis.

The conclusion that mtDNA mutations can be irrelevant to the tumor even if it contains them at high concentration does not mean that mtDNA mutations cannot play important roles in carcinogenesis. Indeed, the number of studies supporting involvement of mtDNA mutations in cancer has

\footnotetext{
Accepted for publication September 11, 2014.

Supported by the Ellison Medical Foundation award (K.K.) and Novartis grant no. 14B065 (K.P.)

Disclosures: K.P. receives funding from Novartis.

Address correspondence to Konstantin Khrapko, Ph.D., 134 Mugar, Department of Biology, Northeastern University, 360 Huntington Ave, Boston, MA 02115 E-mail: k.khrapko@neu.edu
} 
increased in the recent years. It appears, however, that mtDNA mutations can both facilitate and impede carcinogenesis. Initially, positive effects of mtDNA mutations on carcinogenesis were discovered, such as down-regulation of apoptosis by mutations in ATP synthase ${ }^{6}$ or stimulation of metastatic potential by increased reactive oxygen species levels associated with mutations in ND genes. ${ }^{7,8}$ On the other side, it has been argued that functional mitochondrial respiratory chain is indispensable for tumor proliferation. ${ }^{9}$ Furthermore, inactivating ND mutations appear to enhance carcinogenesis at low fractions, but they suppress it once mutations approach homoplasmy. ${ }^{10,11}$ This likely occurs via destabilization of hypoxia-inducible factor $1-\alpha$, which is considered one of the master regulators of the metabolic adaptation needed for malignancy. ${ }^{11}$

\section{Comparison of the Distributions of mtDNA Mutations Can Reveal their Roles in Carcinogenesis}

As argued above, high incidence or high fraction of mtDNA mutations in tumors do not prove that mutations are involved in carcinogenesis. Fortunately, more detailed analysis, such as comparison of mutation frequency distributions by type, position, etc., can help. Indeed, the nonsynonymous to synonymous mutation ratios, or relative frequencies of mutations in one gene versus another, can be changed by selective pressures only. For example, Khajdakov et al ${ }^{12}$ revealed that the proportion of nonsynonymous somatic mtDNA mutations in normal tissues was higher than in tumors. The most natural explanation of this observation is that nonsynonymous mtDNA mutations in tumors are under negative selective pressure, that they impede carcinogenesis. This is an interpretation consistent with other studies. ${ }^{10,11,13}$

Alternately, instead of fewer nonsynonymous mutations in tumors, there may be more of them in normal tissues in comparison to the distribution of de novo generated somatic mutations. Once generated, nascent mutations are subject to positive or negative selective pressures, which change the nonsynonymous to synonymous ratio. From this perspective, higher frequency of nonsynonymous mutations in normal tissues may result either from negative selection in tumors, or from positive selection in normal tissues, or both. Note that positive selection of detrimental mtDNA mutations with age is a well-known phenomenon. ${ }^{14}$ Additional experiments are needed to determine which of the two scenarios is correct, and to establish the distribution of nascent mutations, which is technically difficult. ${ }^{15}$ Whatever the direction of this effect, the difference in nonsynonymous to synonymous ratio of mtDNA mutations between tumors and normal tissue strongly endorses their involvement in carcinogenesis.

Similar to the difference in the proportion of nonsynonymous mutations, a significant excess of mtDNA mutations in one gene compared to another, in tumors but not in normal cells, would strongly support their involvement in carcinogenesis.
Such unequal distributions of mtDNA mutations are indeed observed in tumors, most notably in the oncocytic tumors.

\section{Dissimilar Mutational Distributions in Oncocytic Tumors versus Benign Lesions}

Oncocytic tumors form in various tissues, including the parathyroid gland. Oncocytic tumors originate from precursor cells, which have already undergone oncocytic transformation. mtDNA of many oncocytic tumors have been sequenced and tumor-specific mtDNA mutations determined. ${ }^{16}$ Very notably, the resulting distribution is heavily dominated by mutations in ND genes and just as notably depleted of COX mutations. Specifically, among 67 nonsynonymous mutations, ${ }^{16}$ only $2(3 \%)$ are located in COX genes, whereas $59(88 \%)$ are located in ND genes. The COXND difference is highly significant: $P<0.0001$, Fisher's exact test, odds ratio approximately 10 . This estimate considers that COX and ND genes comprise about $20 \%$ and $50 \%$ of the protein coding part of the mitochondrial genome, respectively.

Such a dramatic disproportion between ND and COX genes implies that mtDNA mutations are important for pathogenesis of oncocytic tumors, but it is not clear yet at what stage they are involved. One possibility is that ND mutations lead to the oncocytic phenotype, whereas COX mutations do not. Then ND mutations will appear enriched, and/or COX mutations will appear depleted because in this case only oncocytic tissue was included in analysis. A second, more interesting possibility is that ND mutations promote tumor progression, whereas COX mutations do not promote or even impede it. This would also result in ND enrichment and COX depletion, because we look at tumors only. As Pereira et $\mathrm{al}^{16}$ advocated, it would be important to look at mutations in nontumor oncocytic cells to resolve this dilemma, but such data are not yet available.

Müller-Höcker et $\mathrm{al}^{1}$ report, unlike Pereira et al, ${ }^{16}$ that mtDNA mutations in COX genes are rather common, at least in their set of benign oncocytic hyperplastic nodules. Moreover, COX mutations (and also other non-ND point mutations) clearly correlate with the progression of the oncocytic phenotype (clear chief cell-pre-oxyphil cell-oxyphil cell). This suggests that oncocytic phenotype can be caused by other mutations than ND, including COX mutations. If so, the predominance of ND mutations and depletion of COX mutations in oncocytic tumors reported by Pereira et al ${ }^{16}$ should not be related to the oncocytic phenotype per se, but rather to tumor progression. This is consistent with recent work reporting ND mutations in tumor progression. ${ }^{17,18}$ Additionally, the observed severe depletion of COX mutations opens a possibility that COX mutations may be actually selected against in oncocytic tumors (ie, potentially impede tumor progression).

If the above inferences are correct, then the difference between the data collected by Pereira et $\mathrm{al}^{16}$ and Müller-Höcker et $\mathrm{al}^{1}$ could rest in the samples; the samples used by MüllerHöcker et $\mathrm{al}^{1}$ were more benign than tumorous samples used 
by Pereira et al. ${ }^{16}$ Indeed, the areas analyzed by Müller-Höcker et $\mathrm{al}^{1}$ are quite small (approximately $1 \mathrm{~mm}$ across) and are explicitly classified as benign, though rigorous comparison of pathology is not possible. These inferences will remain highly speculative unless more data on mutations in oncocytic tumors and even more so on nontumor oncocytic cells are available for analysis.

In conclusion, the study by Müller-Höcker et $\mathrm{al}^{1}$ provide a different view on the distribution and role of mtDNA mutations in oncocytic tumors. These data suggest that ND mutations, which are overrepresented in oncocytic tumors, may be essential for tumor progression rather than merely for induction of the oncocytic phenotype. Similarly, COX mutations, which are greatly underrepresented, may impede oncocytic tumor progression.

\section{References}

1. Müller-Höcker J, Schäfer S, Krebs S, Blum H, Zsurka G, Kunz WS, Prokisch H, Seibel P, Jung A: Oxyphil cell metaplasia in the parathyroids is characterized by somatic mitochondrial DNA mutations in ND genes and cytochrome c oxidase activity-impairing genes. Am J Pathol 2014, 184:2922-2935

2. Polyak K, Li Y, Zhu H, Lengauer C, Willson JK, Markowitz SD, Trush MA, Kinzler KW, Vogelstein B: Somatic mutations of the mitochondrial genome in human colorectal tumours. Nat Genet 1998, 20: 291-293

3. Coller HA, Khrapko K, Bodyak ND, Nekhaeva E, Herrero-Jimenez P, Thilly WG: High frequency of homoplasmic mitochondrial DNA mutations in human tumors can be explained without selection. Nat Genet 2001, 28:147-150

4. Nekhaeva E, Bodyak ND, Kraytsberg Y, McGrath SB, van Orsouw NJ, Pluzhnikov A, Wei JY, Vijg J, Khrapko K: Clonally expanded mtDNA point mutations are abundant in individual cells of human tissues. Proc Natl Acad Sci U S A 2002, 99:5521-5526

5. Taylor RW, Barron MJ, Borthwick GM, Gospel A, Chinnery PF, Samuels DC, Taylor GA, Plusa SM, Needham SJ, Greaves LC, Kirkwood TB, Turnbull DM: Mitochondrial DNA mutations in human colonic crypt stem cells. J Clin Invest 2003, 112:1351-1360

6. Shidara Y, Yamagata K, Kanamori T, Nakano K, Kwong JQ, Manfredi G, Oda H, Ohta S: Positive contribution of pathogenic mutations in the mitochondrial genome to the promotion of cancer by prevention from apoptosis. Cancer Res 2005, 65:1655-1663

7. Petros JA, Baumann AK, Ruiz-Pesini E, Amin MB, Sun CQ, Hall J, Lim S, Issa MM, Flanders WD, Hosseini SH, Marshall FF, Wallace DC: mtDNA mutations increase tumorigenicity in prostate cancer. Proc Natl Acad Sci U S A 2005, 102:719-724

8. Ishikawa K, Takenaga K, Akimoto M, Koshikawa N, Yamaguchi A, Imanishi H, Nakada K, Honma Y, Hayashi J-I: ROS-generating mitochondrial DNA mutations can regulate tumor cell metastasis. Science 2008, 320:661-664

9. Schon EA, DiMauro S, Hirano M: Human mitochondrial DNA: roles of inherited and somatic mutations. Nat Rev Genet 2012, 13:878-890

10. Park JS, Sharma LK, Li H, Xiang R, Holstein D, Wu J, Lechleiter J, Naylor SL, Deng JJ, Lu J, Bai Y: A heteroplasmic, not homoplasmic, mitochondrial DNA mutation promotes tumorigenesis via alteration in reactive oxygen species generation and apoptosis. Hum Mol Genet 2009, 18:1578-1589

11. Gasparre G, Kurelac I, Capristo M, Iommarini L, Ghelli A, Ceccarelli C, Nicoletti G, Nanni P, De Giovanni C, Scotlandi K, Betts CM, Carelli V, Lollini PL, Romeo G, Rugolo M, Porcelli AM: A mutation threshold distinguishes the antitumorigenic effects of the mitochondrial gene MTND1, an oncojanus function. Cancer Res 2011, 71:6220-6229

12. Khaidakov M, Shmookler Reis RJ: Possibility of selection against mtDNA mutations in tumors. Mol Cancer 2005, 4:36

13. McMahon S, LaFramboise T: Mutational patterns in the breast cancer mitochondrial genome, with clinical correlates. Carcinogenesis 2014, 35:1046-1054

14. Khrapko K, Turnbull D: Mitochondrial DNA mutations in aging. Prog Mol Biol Transl Sci 2014, 127:29-62

15. Khrapko K, Vijg J: Mitochondrial DNA mutations and aging: devils in the details? Trends Genet 2009, 25:91-98

16. Pereira L, Soares P, Máximo V, Samuels DC: Somatic mitochondrial DNA mutations in cancer escape purifying selection and high pathogenicity mutations lead to the oncocytic phenotype: pathogenicity analysis of reported somatic mtDNA mutations in tumors. BMC Cancer 2012, 12:53

17. Dasgupta S, Soudry E, Mukhopadhyay N, Shao C, Yee J, Lam S, Lam W, Zhang W, Gazdar AF, Fisher PB, Sidransky D: Mitochondrial DNA mutations in respiratory complex-I in never-smoker lung cancer patients contribute to lung cancer progression and associated with EGFR gene mutation. J Cell Physiol 2012, 227:2451-2460

18. Santidrian AF, Matsuno-Yagi A, Ritland M, Seo BB, LeBoeuf SE, Gay LJ, Yagi T, Felding-Habermann B: Mitochondrial complex I activity and NAD $+/ \mathrm{NADH}$ balance regulate breast cancer progression. J Clin Invest 2013, 123:1068-1081 